CONGENITAL HEART DISEASE

\title{
Prevalence and development of additional cardiac abnormalities in 1448 patients with congenital ventricular septal defects
}

\author{
S Glen, J Burns, P Bloomfield
}

Heart 2004;90:1321-1325. doi: 10.1136/hrt.2003.025007

See end of article for authors' affiliations

\section{Correspondence to:}

Dr Stephen Glen, Stirling Royal Infirmary, Livilands, Stirling FK8 2AU, UK; stephen.glen@fvah.scot. nhs.uk

Accepted

16 February 2004

\begin{abstract}
Objective: To determine by modern echocardiographic techniques the prevalence and development of cardiac abnormalities associated with ventricular septal defect (VSD).

Methods: Consecutive patients referred to a tertiary centre for paediatric cardiology and attenders at an adult congenital heart disease clinic had details of clinical outcome prospectively recorded. Patients with VSD in association with conotruncal abnormalities, atrioventricular septal defects, and univentricular heart were not included in the study.

Results: 1448 patients with VSD were assessed between 1991 and 1998. 1127 (78\%) patients had isolated defects; of these $862(76 \%)$ were perimembranous and $265(24 \%)$ were muscular. Of the remainder, 284 (19.6\% of the total population) patients had VSD associated with one other significant cardiac abnormality: $35(2.4 \%)$ with two and two (0.1\%) with three other abnormalities. The most common associated cardiac abnormalities were infundibular pulmonary stenosis (5.8\%), aortic valve prolapse (3.6\%), pulmonary valvar stenosis $(2.7 \%)$, osteum secundum atrial septal defect $(2.2 \%)$, persistent ductus arteriosus (1.9\%), and coarctation of the aorta (1.5\%). In the cohort of 743 patients followed up from birth, cumulative mortality was $4 \%$ by the age of 8 years and most deaths occurred within the first year of life in infants with associated genetic abnormalities such as trisomy 13 or 18 . Of the 594 patients attending the adult congenital clinic with VSD, aortic regurgitation due to aortic root prolapse developed in 45 (7.6\%) patients. Most of these cases were detected before the patient was 30 years old.

Conclusions: In $22 \%$ of patients with congenital VSD there were significant associated cardiac abnormalities. Some of these abnormalities, such as aortic prolapse and regurgitation or infundibular pulmonary stenosis, may develop or progress subsequently and therefore should be sought during the initial assessment and monitored during follow up. Follow up to the age of 30 years allows the detection of most cases of aortic regurgitation. The prognosis from VSD is excellent and the risk of endocarditis in a population informed of the need for antibiotic prophylaxis is small.
\end{abstract}

$\mathrm{V}$ entricular septal defects (VSDs) are the most common group of congenital cardiac malformations and occur in 2-6 in 1000 live births. ${ }^{12}$ The pattern of defect can range from a tiny hole in the muscular septum, with a good chance of spontaneous closure, to complicated lesions requiring open heart surgery. ${ }^{3-6}$ The prognosis of patients with VSD is good; a large study of patients with congenital VSD found $87 \%$ survival at 25 years although there was a small but significant risk of serious arrhythmia and sudden death even among patients with small defects. ${ }^{6}$ Left ventricular function in some patients may be impaired although the mechanism involved is uncertain. ${ }^{7}$

The prevalence of additional cardiac malformations has been defined in selected groups of patients. In 156 infants with defects large enough to consider cardiac surgery, investigation by echocardiography and cardiac catheterisation confirmed the presence of infundibular pulmonary stenosis in $25 \%$, subaortic ridges in $3 \%$, and persistent ductus arteriosus in 15\%. ${ }^{8}$ A study of 207 patients with muscular VSD found an association with coarctation of the aorta in 11 patients (5\%). ${ }^{4}$ Studies of other cardiac abnormalities, such as persistent ductus arteriosus, confirmed the coexistence of VSD (eight of 146 patients in one group, 5\%). ${ }^{9}$ The presence or development of such abnormalities may necessitate cardiac surgery or long term follow up.

The prevalence of additional abnormalities identified by modern ultrasound technology including colour flow Doppler has not been examined prospectively in a large unselected population. Transthoracic echocardiography with Doppler colour flow mapping is a sensitive and specific technique for the confirmation of clinically suspected $\operatorname{VSD}^{2810}$ and the delineation of associated structural abnormalities.

The aim of this study was to examine the prevalence and characteristics of cardiac abnormalities in patients with congenital VSD by using modern echocardiographic techniques and to identify any further structural abnormalities that may develop with age. Patients with conotruncal abnormalities (transposition of the great arteries, tetralogy of Fallot, persistent truncus arteriosus, or double outlet right ventricle), univentricular heart, and atrioventricular septal defects have a different clinical entity with higher morbidity, mortality, and a corresponding need for corrective surgery. They have not been included in this study.

\section{METHODS}

Details were prospectively recorded for consecutive patients referred to a regional centre for paediatric cardiology or associated satellite clinics who were found to have congenital VSD. VSD was diagnosed by clinical examination and confirmed by transthoracic echocardiography including Doppler colour flow mapping. Additional cardiac abnormalities were sought in these patients and recorded if present.

In addition, patients followed up at an adult congenital heart disease clinic with a known diagnosis of VSD were identified. 


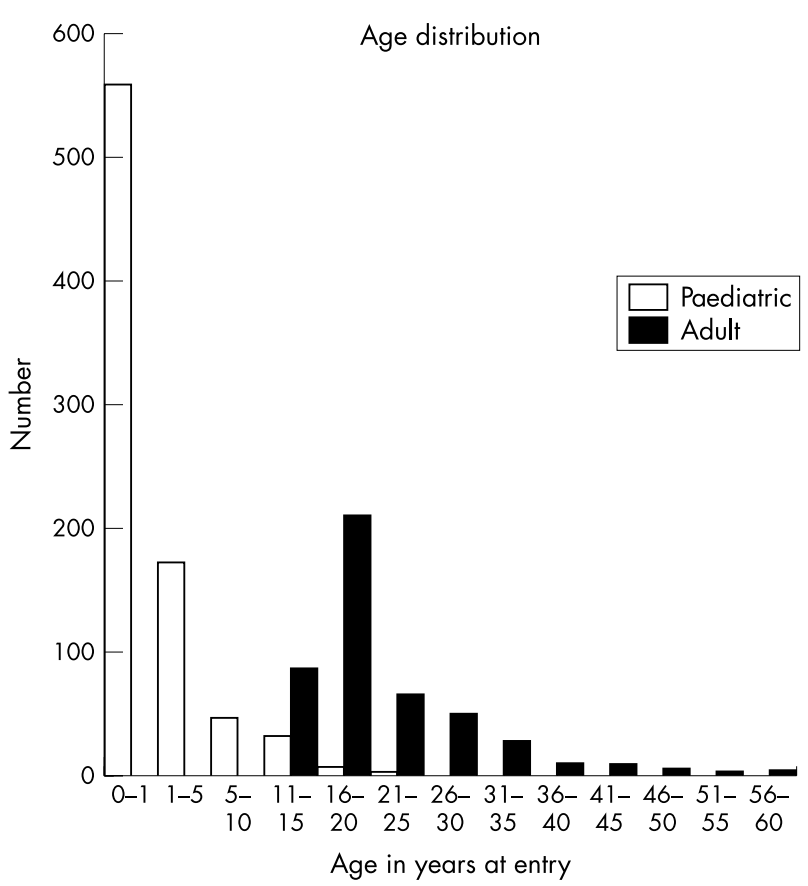

Figure 1 Age distribution of 1448 patients with congenital ventricular septal defect.

Subsequent follow up visits were recorded, as well as details of clinical events including the need for cardiac surgery and cardiovascular complications such as endocarditis and death.

Pulmonary valvar stenosis was diagnosed by echocardiography according to conventional criteria and classified as mild $(<25 \mathrm{~mm} \mathrm{Hg})$, moderate $(25-49 \mathrm{~mm} \mathrm{Hg})$, or severe $(>49 \mathrm{~mm} \mathrm{Hg})$ based on estimated pressure gradients calculated by measurement of flow velocities across the valve. ${ }^{11}$ Infundibular pulmonary stenosis was graded in severity by the same criteria.

Aortic valve prolapse was diagnosed when a deformed aortic valve pivoted from the crest of the interventricular septum, noticeable in the parasternal long axis view. ${ }^{12}$ The degree of aortic regurgitation was defined as trivial if the colour flow regurgitation jet was limited to just below the aortic valve, mild if it did not quite reach the mitral valve tip, moderate if it reached the mitral valve tip, and severe if it was beyond the mitral valve tip. ${ }^{13}$

\section{Statistical analysis}

The $\chi^{2}$ test was used for frequency comparisons between groups and was applied only where more than $20 \%$ of the expected values in the contingency table are greater than 5 . Percentages shown are of the total study population unless otherwise specified. We used SPSS software (version 9.0, SPSS Inc, Chicago, Illinois, USA).

\section{RESULTS}

Between 1991 and 1998, 1448 patients with congenital VSD were identified and assessed by echocardiography. Figure 1 shows the age distribution, which is clearly bimodal, representative of the two patient populations (paediatric and adult groups) studied.

\section{Associated cardiac defects}

Table 1 shows additional associated cardiac defects in order of frequency for new paediatric referrals and adults seen at the adult congenital clinic. When all patients were considered

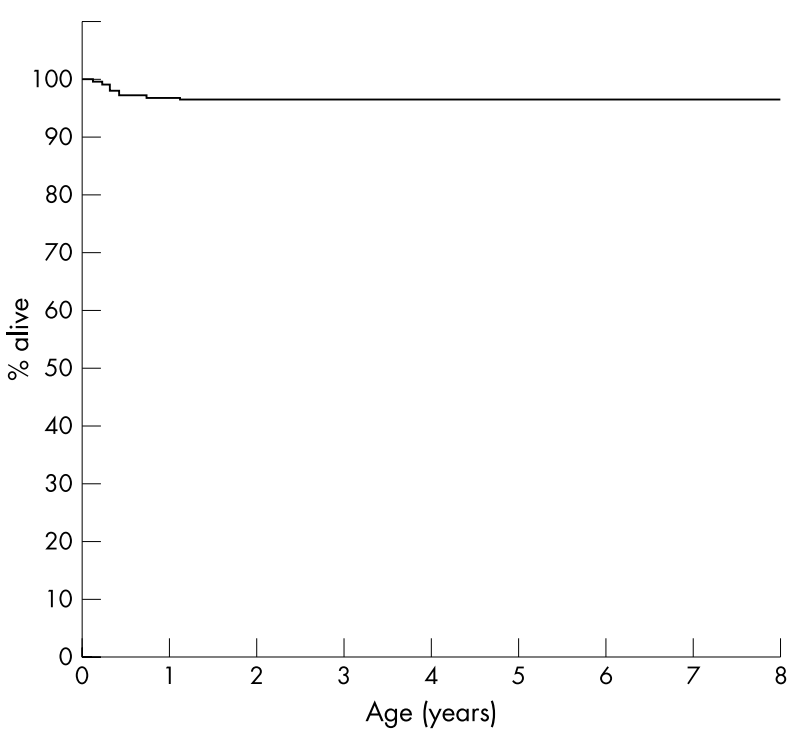

Number available for analysis at each time point (age in years): $\begin{array}{lllllllll}743 & 699 & 638 & 559 & 474 & 410 & 327 & 247 & 153\end{array}$

Figure 2 Kaplan-Meier survival curve for new patients followed up since birth.

together the most commonly associated cardiac abnormalities were infundibular pulmonary stenosis $(5.8 \%)$, aortic prolapse $(3.6 \%)$, pulmonary valvar stenosis $(2.7 \%)$, osteum secundum atrial septal defect $(2.2 \%)$, persistent ductus arteriosus $(1.9 \%)$, and coarctation of the aorta $(1.5 \%)$. The latter three defects listed were significantly less frequent in the adult group because most had been corrected in childhood by surgery or percutaneous intervention. Aortic valve prolapse was more frequent in the adult group $(6.7 \% v 1.3 \%$, $\mathrm{p}<0.0001)$, as was infundibular pulmonary stenosis $(7.8 \% \mathrm{v}$ $4.3 \%, \mathrm{p}<0.005)$.

The majority of VSDs occurred in isolation but in $19.6 \%$ of patients they were associated with one other cardiac abnormality, in $2.4 \%$ with two other abnormalities, and in only $0.1 \%$ with three abnormalities (table 2 ). Muscular defects were less common in the adult group as would be expected due to spontaneous closure in childhood and adolescence.

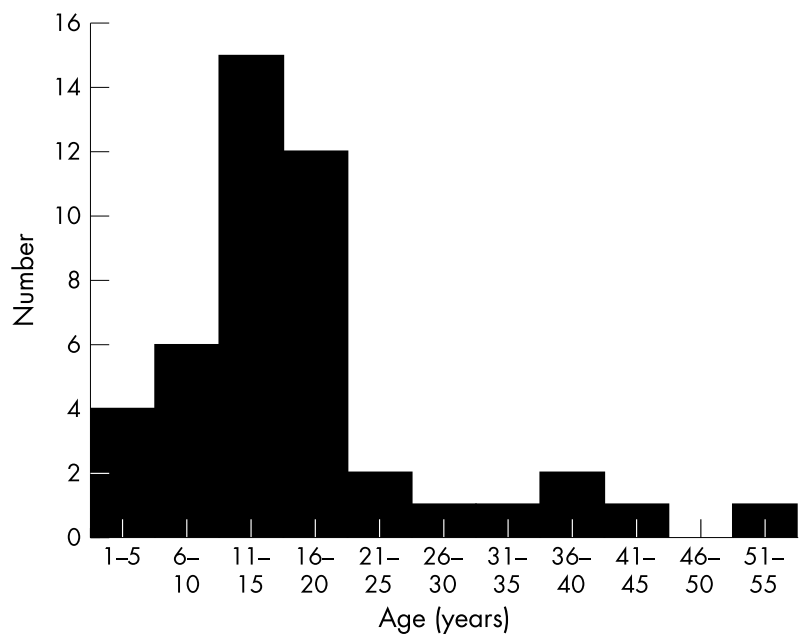

Figure 3 Age when aortic regurgitation first detected. 
Table 1 Prevalence of additional cardiac abnormalities in patients with ventricular septal defect (VSD) with a comparison of frequencies between the adult and paediatric groups

\begin{tabular}{lllll}
\hline & Group & & & \\
\cline { 2 - 4 } & All & Paediatric & Adult & p Value \\
\hline IPS & $84(5.80 \%)$ & $36(4.31 \%)$ & $48(7.84 \%)$ & $<0.005$ \\
Aortic prolapse & $52(3.59 \%)$ & $11(1.32 \%)$ & $41(6.70 \%)$ & $<0.0001$ \\
Pulmonary valvar stenosis & $39(2.69 \%)$ & $25(2.99 \%)$ & $14(2.29 \%)$ & NS \\
Osteum secundum ASD & $32(2.21 \%)$ & $23(2.75 \%)$ & $9(1.47 \%)$ & NS \\
PDA & $27(1.86 \%)$ & $24(2.87 \%)$ & $3(0.49 \%)$ & $<0.001$ \\
CoA & $22(1.52 \%)$ & $20(2.39 \%)$ & $2(0.33 \%)$ & $<0.005$ \\
Osteum secundum ASD and PDA & $13(0.90 \%)$ & $12(1.44 \%)$ & $1(0.16 \%)$ & $<0.05$ \\
Mitral regurgitation & $12(0.83 \%)$ & $5(0.60 \%)$ & $7(1.14 \%)$ & NS \\
IPS and aortic prolapse & $8(0.55 \%)$ & $3(0.36 \%)$ & $5(0.82 \%)$ & NS \\
Wolff-Parkinson-White syndrome & $5(0.35 \%)$ & $3(0.36 \%)$ & $2(0.33 \%)$ & NS \\
Aortic valvar stenosis & $3(0.21 \%)$ & $2(0.24 \%)$ & $1(0.16 \%)$ & NS \\
Pulmonary valvar stenosis and PDA & $3(0.21 \%)$ & $3(0.36 \%)$ & 0 & NA \\
PBS & $3(0.21 \%)$ & 0 & $3(0.49 \%)$ & NA \\
CoA and IPS & $2(0.14 \%)$ & $2(0.24 \%)$ & 0 & NA \\
Aortic valvar stenosis and CoA & $2(0.14 \%)$ & $2(0.24 \%)$ & 0 & NA \\
IPS and osteum secundum ASD & $2(0.14 \%)$ & $1(0.12 \%)$ & $1(0.16 \%)$ & NA \\
PBS and ASD & $2(0.14 \%)$ & $1(0.12 \%)$ & $1(0.16 \%)$ & NA \\
Mitral stenosis & $2(0.14 \%)$ & 0 & $2(0.33 \%)$ & NA \\
Dextrocardia & $2(0.14 \%)$ & 0 & $2(0.33 \%)$ & NA \\
Aortic prolapse and IPS and CoA & $1(0.07 \%)$ & $1(0.12 \%)$ & 0 & NA \\
Pulmonary supravalvar stenosis & $1(0.07 \%)$ & $1(0.12 \%)$ & 0 & NA \\
Aortic valvar stenosis and PDA & $1(0.07 \%)$ & $1(0.12 \%)$ & 0 & NA \\
Mitral stenosis and CoA & $1(0.07 \%)$ & $1(0.12 \%)$ & 0 & NA \\
PDA and CoA & $1(0.07 \%)$ & $1(0.12 \%)$ & 0 & NA \\
IPS and PDA and aortic stenosis & $1(0.07 \%)$ & $1(0.12 \%)$ & 0 & NA \\
\hline
\end{tabular}

ASD, atrial septal defect; CoA, coarctation of the aorta; IPS, infundibular pulmonary stenosis; NA, not applicable; NS, not significant; PBS, pulmonary branch stenosis; PDA, persistent ductus arteriosus.

\section{Follow up}

In their first year of life 743 patients were assessed. Figure 2 shows eight years of prospective clinical follow up with $4 \%$ cumulative all cause mortality (29 deaths) by the age of 8 years. The majority of deaths occurred during the first year in infants with additional genetic abnormalities as table 3 and table 4 show. No patient developed endocarditis in the follow up cohort. In addition, 16 patients were assessed in the first year of life but were then lost to follow up and have not been included in this analysis. Eleven of these patients had small muscular defects.

Figure 3 shows an increase in the number of patients with aortic regurgitation with age with a peak between 11 and 15 years of age.

Overall 105 patients in the adult group required corrective surgery (including percutaneous closure) although in most cases this was performed in childhood with a mean (SD) age of 4.7 (4.7) years at the time of surgery. During the prospective follow up of the paediatric group 106 patients required corrective surgery $(12.6 \%)$, and this was performed at a mean (SD) age of $1.4(2.1)$ years.

Of the 15 paediatric patients with aortic valve prolapse, moderate or severe regurgitation was detected in five (33\%). Of the 46 adult patients with aortic valve prolapse, moderate or severe regurgitation was apparent in five (all of whom underwent surgery with aortic valve replacement in three and cusp resuspension in two) and 19 patients had trivial or mild aortic regurgitation.

\section{DISCUSSION}

We have found that about $20 \%$ of patients with congenital VSD have additional cardiac abnormalities. Most abnormalities were detected at the initial assessment but aortic prolapse and infundibular pulmonary stenosis may develop subsequently. Such abnormalities may influence management, although the overall prognosis from VSD was excellent in this population and the risk of endocarditis was low.

Secondary aortic regurgitation may result from the venturi effect of high velocity flow beneath an inadequately supported right aortic valve cusp ${ }^{14}$ or following maldevelopment of the aortic commissure. Continuing clinical follow up is therefore required for patients with VSD beyond adolescence and in our study $82 \%$ of cases of secondary aortic regurgitation were detected before the age of 20 years. Extending the follow up of patients to the age of 30 would allow the detection of $89 \%$ of patients with secondary aortic regurgitation. The indications for aortic valve surgery in aortic root prolapse are controversial. Recent evidence

Table 2 Association of VSD with other cardiac abnormalities

\begin{tabular}{llll}
\hline & \multicolumn{2}{l}{ Number of defects } & \\
\cline { 2 - 4 } & Paediatric group & Adult group & Overall \\
\hline Isolated VSD & $657(78.6 \%)$ & $470(76.8 \%)$ & $1127(77.8 \%)$ \\
$\quad$ Perimembranous & $461(55.1 \%)$ & $401(65.5 \%)$ & $862(59.5 \%)$ \\
$\quad$ Muscular & $196(23.4 \%)$ & $69(11.3 \%)$ & $265(18.3 \%)$ \\
One associated abnormality & $150(17.9 \%)$ & $134(21.9 \%)$ & $284(19.6 \%)$ \\
Two associated abnormalities & $27(3.2 \%)$ & $8(1.3 \%)$ & $35(2.4 \%)$ \\
Three associated abnormalities & $2(0.2 \%)$ & 0 & $2(0.1 \%)$ \\
Total & 836 & 612 & 1448 \\
\hline
\end{tabular}


Table 3 Deaths in the follow up cohort

\begin{tabular}{|c|c|c|}
\hline $\begin{array}{l}\text { Age at death } \\
\text { (years) }\end{array}$ & Cardiac abnormality & Additional abnormality \\
\hline 0.01 & Isolated VSD & \\
\hline 0.01 & Isolated VSD & Trisomy 18 \\
\hline 0.02 & VSD and secundum ASD & Trisomy 18 \\
\hline 0.03 & Isolated VSD & Trisomy 13 \\
\hline 0.04 & Isolated VSD & Preterm infant, necrotising enterocolitis \\
\hline 0.06 & Isolated VSD & Dysmorphic, Hirschhorn \\
\hline 0.08 & Isolated VSD & Trisomy 13 \\
\hline 0.08 & Isolated VSD & Trisomy 18 \\
\hline 0.09 & Isolated VSD & Trisomy 13 \\
\hline 0.16 & Isolated VSD & Down's syndrome, duodenal atresia \\
\hline 0.23 & VSD, ASD, PFO, and left PBS & \\
\hline 0.25 & Isolated VSD & $22 q 11$ deletion syndrome \\
\hline 0.27 & VSD and aortic incompetence & Immotile cilia \\
\hline 0.29 & VSD and secundum ASD & \\
\hline 0.30 & Isolated VSD & \\
\hline 0.31 & VSD and IPS & \\
\hline 0.33 & Isolated VSD & \\
\hline 0.37 & Isolated VSD & \\
\hline 0.37 & Isolated VSD & Cleft lip, palate, pseudotrisomy 18 \\
\hline 0.38 & Isolated VSD & Down's syndrome, preterm \\
\hline 0.41 & Isolated VSD & Preterm infant \\
\hline 0.41 & Isolated VSD & Down's syndrome (preterm infant) \\
\hline 0.44 & Isolated VSD & \\
\hline 0.49 & VSD and PDA & Multiple congenital abnormalities \\
\hline 0.64 & Isolated VSD & Trisomy 18 \\
\hline 0.74 & Isolated VSD & Holt-Oram syndrome \\
\hline 0.85 & Isolated VSD & Trisomy 18 \\
\hline 0.87 & Isolated VSD & \\
\hline
\end{tabular}

suggests that the progression from mild to severe incompetence may be rapid and that surgical intervention should be early. ${ }^{15}$

Echocardiography with colour flow mapping is an effective technique for the detection of $\mathrm{VSD}^{16}$ and in fact is more sensitive than cardiac catheterisation in the detection of multiple septal defects. ${ }^{8}$ Echocardiography has also been shown to be as sensitive as cardiac catheterisation in the detection of other cardiac abnormalities, such as infundibular pulmonary stenosis. ${ }^{8}$ Echocardiography continues to develop with advances such as harmonic imaging ${ }^{17}$ and transducer characteristics $^{18}$ that allow higher frequency imaging and improved image quality. Colour flow Doppler processing has been the most important advance in the detection of VSD, as flow across the interventricular septum can be easily detected. ${ }^{19}$ Although other techniques are evolving such as the use of contrast agents, ${ }^{20}$ three dimensional echocardiography, ${ }^{21} 22$ and magnetic resonance imaging, ${ }^{23}$ it seems unlikely that these advances will significantly increase the confirmation rate of clinically suspected VSD or of associated cardiac abnormalities.

This study was not primarily designed to measure long term clinical outcome and all cause mortality was recorded to ensure that cases were not missed because of misclassification of death. The prevalence statistics of associated cardiac abnormalities reflects true clinical practice, as patients were assessed consecutively without selection bias.

Table 4 Chromosomal abnormalities in the paediatric group

\begin{tabular}{lc}
\hline Chromosomal abnormality & Number of patients \\
\hline Trisomy 21 & $29(3.5 \%)$ \\
Trisomy 18 & $7(0.8 \%)$ \\
Trisomy 13 & $4(0.5 \%)$ \\
22 11 deletion & $4(0.5 \%)$ \\
\hline
\end{tabular}

\section{Summary}

Echocardiography confirms the presence of additional cardiac abnormalities in $22 \%$ of patients with congenital VSD. These should be sought during the initial assessment, and the subsequent development of aortic prolapse or infundibular pulmonary stenosis should be monitored during clinical follow up. Follow up to the age of 30 years allows the detection of most but not all cases of secondary aortic regurgitation.

\section{Authors' affiliations}

S Glen, P Bloomfield, Royal Infirmary of Edinburgh, Edinburgh, UK

J Burns, Royal Hospital for Sick Children, Edinburgh, UK

\section{REFERENCES}

1 Samanek M, Voriskova M. Congenital heart disease among 815,569 children born between 1980 and 1990 and their 15 year survival: a prospective Bohemia survival study. Pediatr Cardiol 1999;20:411-7.

2 Meberg A, Otterstad JE, Froland G, et al. Increasing incidence of ventricular septal defects caused by improved detection rate. Acta Paediatr 1994:83:653-7.

3 Mehta AV, Chidambaram B. Ventricular septal defect in the first year of life. Am J Cardiol 1992;70:364-6.

4 Ramaciotti C, Vetter JM, Bornemeier RA, et al. Prevalence, relation to spontaneous closure, and association of muscular ventricular septal defects with other cardiac defects. Am J Cardiol 1995;75:61-5.

5 Frontera-Izquierdo $P$, Cabezuelo-Huerta G. Natural and modified history of isolated ventricular septal defect: a 17-year study. Paediatr Cardiol 1992:13:193-7.

6 Kidd L, Driscoll DJ, Gersony WM, et al. Second natural history study of congenital heart defects: results of treatment of patients with ventricular septal defects. Circulation 1993;87(suppl I):138-51.

7 Magee AG, Fenn L, Vellekoop J, et al. Left ventricular function in adolescents and adults with restrictive ventricular septal defect and moderate left-to-right shunting. Cardiol Young 2000;10:126-9.

8 Magee AG, Boutin C, McCrindle BW, et al. Echocardiography and cardiac catheterisation in the preoperative assessment of ventricular septal defect in infancy. Am Heart J 1998; 135:907-13.

9 Gelb BD, O'Laughlin MP, Mullins CE. Prevalence of additional cardiovascular anomalies in patients referred for transcatheter closure of patent ductus arteriosus. J Am Coll Cardiol 1990;16:1680-6.

10 Sutherland GR, Godman MJ, Smallhorn JF, et al. Ventricular septal defects: two dimensional echocardiographic and morphological correlations. $\mathrm{Br}$ Heart J 1982;47:316-28. 
11 O'Fallon WM, Weidman WH, eds. Long-term follow-up of congenital aortic stenosis, pulmonary stenosis, and ventricular septal defect: report from the second joint study of congenital heart defects (NHS-2). Circulation 1993;87(suppl I):11-126.

12 Craig BG, Smallhorn JF, Burrows P, et al. Cross-sectional echocardiography in the evaluation of aortic valve prolapse associated with ventricular septal defects. Am Heart J 1986; 112:800-7.

13 Tohyama K, Satomi G, Momma K. Aortic valve prolapse and aortic regurgitation associated with subpulmonic ventricular septal defect. Am J Cardiol 1997:79:1285-9.

14 Ando M, Takao A. Pathological anatomy of ventricular septal defect associated with aortic valve prolapse and regurgitation. Heart Vessels 1986;2:117-26.

15 Eroglu AG, Oztunc F, Saltik L, et al. Aortic valve prolapse and aortic regurgitation in patients with ventricular septal defect. Pediatr Cardiol 2003:24:36-9.

16 Helmcke F, de Souza A, Nanda NC, et al. Two-dimensional and color Doppler assessment of ventricular septal defect of congenital origin. Am J Cardiol 1989;63:1112-6.
17 Caidahl K, Kazzam E, Lidberg J, et al. New concept in echocardiography: harmonic imaging of tissue without use of contrast agent. Lancet 1998;352:1264-70.

18 Marx GR. Advances in cardiac imaging in congenital heart disease. Curr Opin Pediatr 1995:7:580-6.

19 Ritter SB. Two-dimensional Doppler colour flow mapping in congenital heart disease. Clin Cardiol 1986;9:591-6.

$20 \mathrm{Kaul} \mathrm{S.} \mathrm{New} \mathrm{developments} \mathrm{in} \mathrm{ultrasound} \mathrm{systems} \mathrm{for} \mathrm{contrast}$ echocardiography. Clini Cardiol 1997;20(10 suppl 1):127-30.

21 De Castro S, Yao J, Pandian NG. Three-dimensional echocardiography: clinical relevance and application. Am J Cardiol 1998;81: 96G-102G.

22 Dall'Agata A, Cromme-Dijkhuis AH, Meijboom FJ, et al. Three-dimensional echocardiography enhances the assessment of ventricular septal defect. Am J Cardiol 1999:83:1576-9.

23 Didier D, Higgins CB. Identification and localization of ventricular septal defect by gated magnetic resonance imaging. Am J Cardiol 1986;57:1363-8.

\section{IMAGES IN CARDIOLOGY}

\section{Quadri-leaflet pulmonary valve: unusual cause of unexplained murmur}

doi: 10.1136/hrt.2004.034181

A 43 year old woman was referred for cardiac magnetic resonance (CMR) imaging for investigation of a systolic murmur.

The patient was asymptomatic with no previous episodes of chest pain, breathlessness, palpitations, or syncope. Her exercise tolerance was good. There were no risk factors for ischaemic heart disease, no history of hypertension or diabetes, and she had never smoked.

On examination she was well with no jaundice, cyanosis, anaemia, or clubbing. She was normotensive and in sinus rhythm. The jugular venous pressure (JVP) was not elevated. On auscultation she had a loud pansystolic murmur audible throughout the precordium but did not radiate elsewhere. There were no carotid bruits.

Her resting ECG was normal as was a recent computed tomographic (CT) chest scan. Her echocardiogram was reported as normal. The murmur was therefore assumed to be "innocent" but the patient was referred to CMR Unit, Royal Brompton Hospital, to rule out a small ventricular septal defect (VSD).
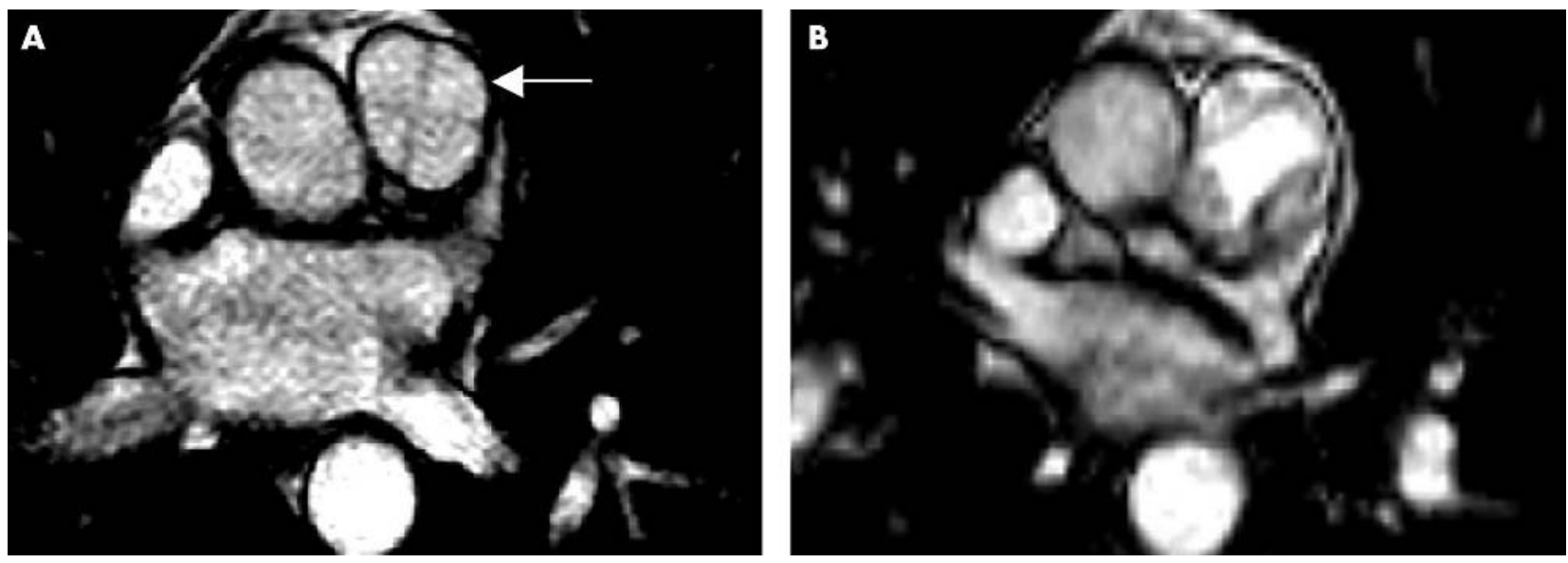

Selected cine images in the short axis of the pulmonary valve acquired in diastole (A) and systole (B), showing a four leaflet pulmonary valve (arrow) and a rectangular orifice.
We performed standard multislice acquisitions in all tomographic planes and cine imaging of cardiac structures in their long and short axes. Cine imaging revealed an area of turbulence emanating from the pulmonary valve. Further cine imaging with and without flow mapping (panel below) revealed a four leaflet pulmonary valve which was not significantly stenosed (peak velocity $1.6 \mathrm{~m} / \mathrm{s}$ ). Mild pulmonary valve regurgitation was noted. Abnormalities of the pulmonary valve are relatively common.

There are few previous reports of a four leaflet pulmonary valve but, to our knowledge, this is the first report demonstrated by CMR. The systolic murmur auscultated is presumed to arise from the increased turbulence generated by disturbed flow through this valve and its close proximity to the anterior chest wall.

G C Smith

R H Mohiaddin r.mohiaddin@rbh.nthames.nhs.uk 